\title{
Conhecimentos, preocupações e atitudes dos pais perante a febre
}

Ivete Afonso, ${ }^{1}$ Sofia Faria, ${ }^{2}$ Sílvia Martins, ${ }^{2}$ Carla Silva, ${ }^{2}$ Hugo Rocha, ${ }^{2}$ Raquel Braga ${ }^{3}$

\section{RESUMO}

Objetivos: Caracterizar os conhecimentos, preocupações e atitudes dos pais perante uma criança com febre, de forma a traçar um plano futuro de intervenção nesta área junto da população.

Tipo de estudo: Estudo transversal, descritivo e analítico.

Local: USF Lagoa.

População: Todos os pais ou cuidadores de crianças com idades entre os 29 dias e os 36 meses inscritos na USF Lagoa.

Métodos: Estudo realizado entre setembro e dezembro de 2015, através da aplicação de um questionário por via telefónica ou diretamente com o cuidador, caso este tivesse consulta agendada durante o mesmo período de tempo. Foi feita uma análise descritiva simples, com recurso ao programa Microsoft Excel $2010^{\circledR}$. Este estudo é reportado de acordo com as linhas de orientação STROBE.

Resultados: Foram entrevistados 248 pais/cuidadores. Oitenta e três por cento fazem avaliação da temperatura na axila e $40 \%$ considera febre para valores inferiores a $37,5^{\circ} \mathrm{C}$. A maioria $(54,7 \%)$ escolhe como principal fármaco a administrar o paracetamol e $30 \%$ sabe administrar doses corretas de medicação. As convulsões são consideradas a principal complicação associada à febre $(62,1 \%)$. Cinquenta por cento recorre ao médico logo que surge febre ou no primeiro dia de doença, sendo o centro de saúde o local de eleição para a metade dos inquiridos.

Conclusões: Perceções e atitudes erradas em relação à febre persistem nos dias de hoje. É fundamental reforçar os conhecimentos dos pais/cuidadores em relação à abordagem e tratamento da febre, de forma a diminuir a ansiedade e melhorar a prestação de cuidados à criança febril.

Palavras-chave: Antipiréticos; Febre; Informação em saúde

\section{INTRODUÇÃO}

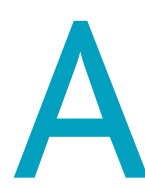

febre é um dos sinais de doença mais frequentes na criança/adolescente. ${ }^{1}$ É ainda o motivo mais frequente de procura de cuidados de saúde em idade pediátrica, representando cerca de $20 \%$ das causas de recurso aos serviços de urgência hospitalares. ${ }^{1}$

Historicamente, a falta de informação relativamente a este tema, nomeadamente o pressuposto de que a temperatura não controlada aumenta progressivamente e, desta forma, causa dano neurológico, ou mesmo a morte, deu origem a uma «fobia», descrita por

1. Médica Interna de Pediatria. ULS Matosinhos, Hospital Pedro-Hispano 2. Médico Interno de Medicina Geral e Familiar. ULS Matosinhos, USF Lagoa 3. Assistente graduada em Medicina Geral e Familiar. ULS Matosinhos, USF Lagoa
Schmitt em $1980 .^{2}$ No entanto, esta «fobia» continua a ser sentida em todos os estratos socioeconómicos e culturais, bem como a necessidade de combater a febre mediante a utilização excessiva de antipiréticos, com efeitos potencialmente tóxicos. ${ }^{1,3-4}$

Tendo em consideração a elevada procura de serviços de saúde, é de extrema importância a existência de estudos que investiguem as principais preocupações dos cuidadores em relação a este sintoma, os seus conhecimentos, as atitudes que consideram adequadas perante uma intercorrência febril e a forma como administram antipiréticos.

\section{MÉTODOS}

Foi conduzido um estudo transversal, descritivo e analítico através da aplicação de um questionário por 
via telefónica ou diretamente ao pai/cuidador, caso este tivesse consulta agendada com o seu filho no mesmo período de tempo. Este foi dirigido aos pais ou cuidadores de crianças com idades entre os 29 dias e os 36 meses inscritos na Unidade de Saúde Familiar Lagoa.

O questionário foi concebido pelos autores e aplicado após realização de um estudo piloto. O questionário (Apêndice A) era composto por 19 perguntas de escolha simples (as várias opções apresentadas eram apenas visíveis para o investigador, de forma a facilitar a codificação posterior). As perguntas A e B referiam-se aos dados do lactente (data de nascimento e peso) e as perguntas C-F aos dados do principal cuidador (grau de parentesco, idade, habilitações literárias e existência de mais filhos). As perguntas G-I eram relativas à avaliação da temperatura (como, em que local, valor a partir do qual é considerado febre); as questões J-M incidiam sobre a administração de antipiréticos (administração prévia ao recurso a serviços de saúde, valor a partir do qual é administrada terapêutica); a pergunta M questionava outras atitudes a ter relativamente à febre; as questões $\mathrm{O}$ e $\mathrm{P}$ reportavam-se à utilização dos serviços de saúde (em que dia de febre e onde); as questões Q e $\mathrm{R}$ aludiam aos conhecimentos relativos à febre $\mathrm{e}$, finalmente, a questão $S$ referia-se à abordagem prévia do assunto.

A recolha dos dados foi realizada entre setembro e dezembro de 2015.

Foram incluídos todos os utentes inscritos que concluíram o questionário, sendo considerada apenas uma participação caso o cuidador tivesse mais do que um filho incluído na amostra. Foram excluídos todos os utentes que, apesar de cumprirem critérios, não apresentassem registo de número de telefone no processo clínico ou caso o mesmo não fosse válido.

Os dados foram submetidos a uma análise descritiva, com recurso ao programa Microsoft Excel 2010®. Foram utilizadas as linhas de orientação Strengthening the Reporting of Observational Studies in Epidemiology (STROBE) para a elaboração do presente artigo.

A realização deste estudo foi aprovada pelos presidentes dos conselhos clínicos e de administração do ACeS de Matosinhos, tendo merecido parecer favorável por parte da comissão de ética da ULS Matosinhos. Todos os participantes deram o seu consentimento de forma informada.

\begin{tabular}{|lc|}
\hline \multicolumn{2}{|l|}{ QUADRO I. Escolaridade dos pais/cuidadores } \\
\hline Habilitações & $n(\%)$ \\
\hline Ensino básico/primário & $50(20,2)$ \\
\hline Ensino secundário & $82(33,1)$ \\
\hline Licenciatura & $102(41,1)$ \\
\hline Mestrado/Doutoramento & $14(5,6)$ \\
\hline Total & $248(100)$ \\
\hline
\end{tabular}

Legenda: $n$ = número; \% = percentagem

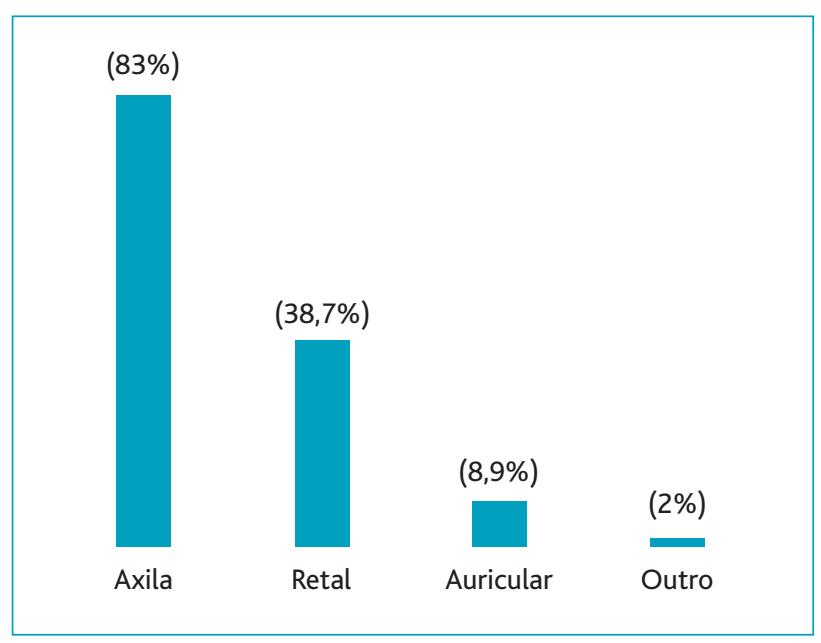

Figura 1. Local de avaliação da temperatura corporal pelos pais/cuidadores.

Legenda: \% = percentagem

\section{RESULTADOS}

Aceitaram participar no estudo 248 pais/cuidadores dos 310 lactentes e crianças inscritos, os quais forneceram o seu consentimento informado. A taxa de participação foi de $80 \%$. A idade dos pais variou entre 18 e 46 anos (média 32,8 anos) e a dos outros cuidadores entre 37 e 73 anos (média 60,5 anos). A mãe foi a principal inquirida (87,9\%). Relativamente às habilitações, $41 \%$ era licenciado e $20 \%$ da população inquirida possuía o ensino básico. A distribuição do nível de escolaridade dos pais/cuidadores encontra-se representado no Quadro I. A média de idades das crianças avaliadas foi de 21 meses (1 mês - 35 meses). Relativamente à forma de avaliação da temperatura, a maioria (83\%) escolheu a axila como local de avaliação (Figu- 


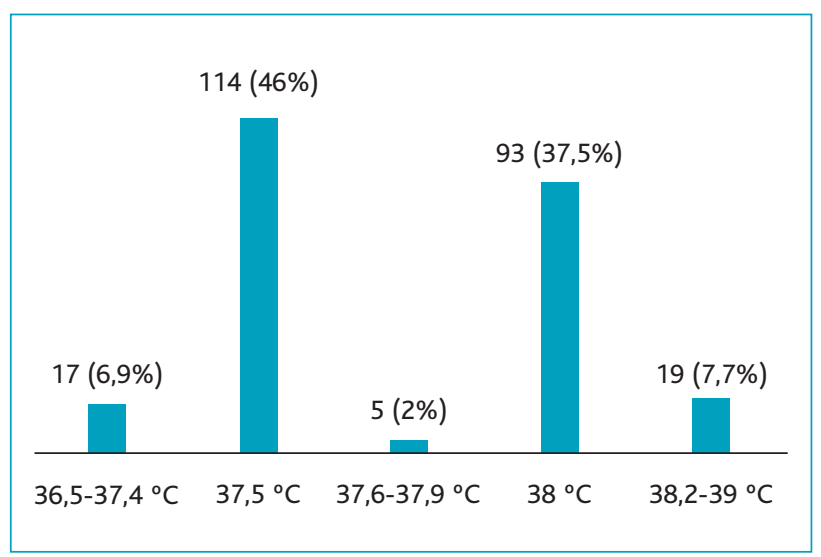

Figura 2. Valor a partir do qual foi considerado febre pelos pais/cuidadores.

Legenda: \% = percentagem

\begin{tabular}{lc} 
QUADRO II. Dose a administrar antipiréticos $(n=248)$ \\
Dose & $n(\%)$ \\
\hline Correta & $66(27)$ \\
\hline Supra-terapêutica & $72(29,4)$ \\
\hline Infra-terapêutica & $47(18,9)$ \\
\hline Desconhece & $63(25,7)$ \\
\hline Total & $248(100)$
\end{tabular}

Legenda: $n$ = número; $\%$ = percentagem

ra 1). Quarenta e sete por cento consideraram existir febre se a temperatura fosse inferior a $37,5{ }^{\circ} \mathrm{C}$, independentemente do local de avaliação e cerca de $7 \%$ referiu valores para febre entre $36,5^{\circ} \mathrm{C}$ e $37,4{ }^{\circ} \mathrm{C}$ (Figura 2). Quase todos afirmaram ter no domicílio antipiréticos, sendo o fármaco mais utilizado o paracetamol $(54,7 \%)$. No caso de possuírem paracetamol e ibuprofeno, o paracetamol foi o fármaco preferido para administração em primeiro lugar. Vinte e sete por cento sabiam administrar de forma correta a medicação (Quadro II). Dezasseis por cento admitiu não administrar medicação antes do recurso aos serviços de saúde, sendo o medo de mascarar os sintomas a principal razão apontada $(74,4 \%)$. Os restantes afirmaram só administrar medicação após contacto com um médico $(5,1 \%)$ ou não administravam por desconhecer a dose correta a administrar.
Sessenta e dois por cento referiram que as principais complicações da febre não tratada eram as convulsões e, em segundo lugar, a possibilidade de lesão cerebral (20\%), sem conseguirem especificar qual o tipo de lesão. Apenas 2,8\% considerou a possibilidade de desidratação (Figura 3).

Cerca de 50\% admitiu recorrer ao médico logo que surgisse febre ou no primeiro dia de doença. Relativamente ao local escolhido para observação, $50 \%$ optou pelo seu centro de saúde, seguido dos hospitais públi$\cos (21,8 \%)$, privados $(21,8 \%)$, linha de saúde $24(5,6 \%)$ e farmácia $(0,2 \%)$.

\section{DISCUSSÃO}

De uma forma geral, este estudo mostra que o conhecimento dos pais/cuidadores relativamente ao conceito de febre, sua abordagem e consequências é deficitário.

À semelhança do observado noutros estudos, também nesta amostra a maioria dos pais prefere avaliar a temperatura na axila, apesar de estar formalmente estabelecido que a medição retal é a forma recomendada nas crianças com idade inferior a 24 meses, dado ser o método que melhor corresponde à temperatura central..$^{1,4}$

De uma forma geral, pode dizer-se que há febre quando existe uma elevação da temperatura corporal $\geq 1{ }^{\circ} \mathrm{C}$ acima da média diária individual, dependendo da hora e do local de medição. ${ }^{1}$ Não se sabendo a temperatura média diária individual, a mais recente Norma da Direção-Geral da Saúde considera o diagnóstico de febre quando se atinge qualquer dos seguintes valores da temperatura: retal $\geq 38^{\circ} \mathrm{C}$; axilar $\geq 37,6^{\circ} \mathrm{C}$; timpânica $\geq 37,8^{\circ} \mathrm{C}$; oral $\geq 37,6^{\circ} \mathrm{C} .{ }^{1}$ Nesta amostra, tendo em conta o local de avaliação da temperatura mais utilizado pelos pais/cuidadores, é preocupante persistir o conceito de que existe febre se a temperatura corporal for inferior a $37,5^{\circ} \mathrm{C}$. O estabelecimento de temperaturas inferiores a $38^{\circ} \mathrm{C}$ como limiar de tratamento farmacológico foi referido em $40 \%$ das respostas, valor ainda mais elevado em relação ao reportado em estudos prévios. $^{4-6}$

Neste estudo, praticamente a totalidade dos cuidadores possuía medicação no domicílio. Importa salientar que apesar de a maioria reconhecer o paracetamol como fármaco de primeira linha, apenas $30 \%$ afir- 


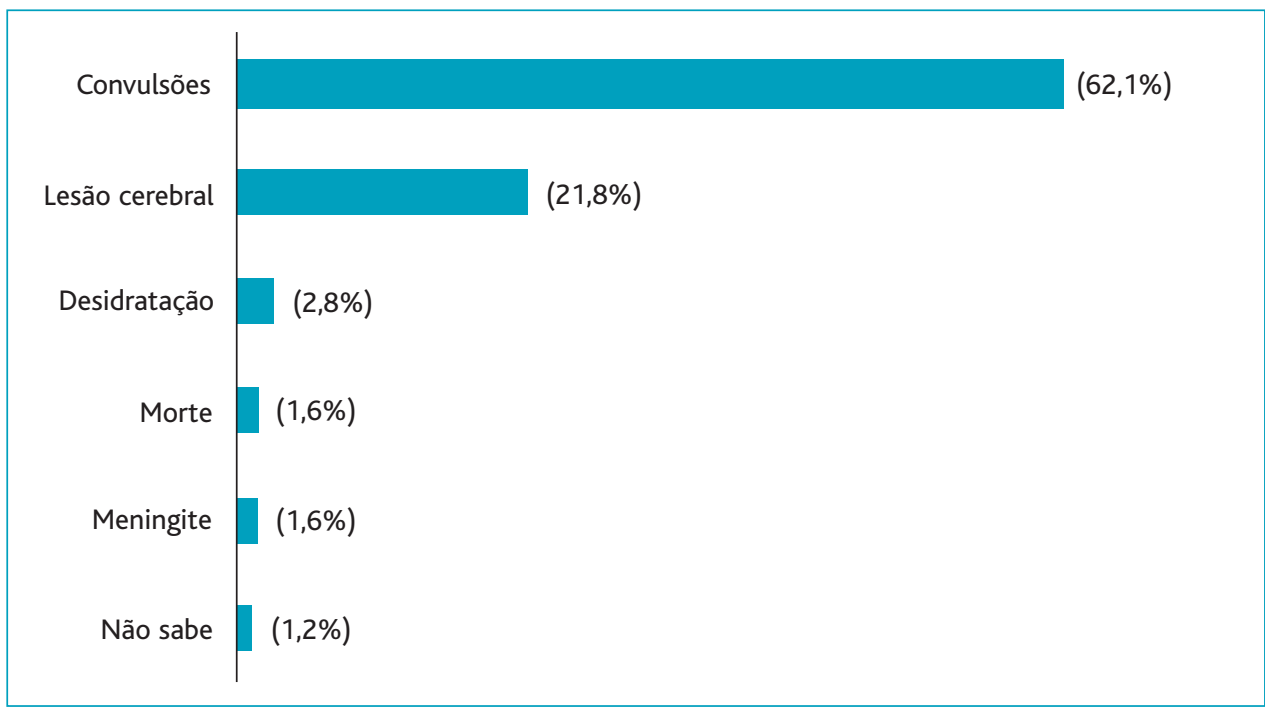

Figura 3. Principais complicações da febre referidas pelos pais/cuidadores. Legenda: \% = percentagem

mava administrar doses corretas. Foi possível avaliar este dado a partir do peso fornecido pelo pai/cuidador ou através da consulta do processo clínico do utente. Foi considerada dose correta de paracetamol oral quando a mesma se situava entre $10-15 \mathrm{mg} / \mathrm{Kg} / \mathrm{dose}$; retal entre $15-20 \mathrm{mg} / \mathrm{Kg} /$ dose e ibuprofeno oral entre $5-10 \mathrm{mg} / \mathrm{Kg} /$ dose. ${ }^{1}$ Nenhum entrevistado admitiu utilizar qualquer outro fármaco. A administração de medicação antes do recurso a um serviço de saúde não foi alvo de avaliação na literatura pesquisada. Neste estudo, dezasseis por cento dos inquiridos refere não administrar medicação antes da avaliação por um médico com medo de «mascarar sintomas» e 50\% recorre ao médico logo que surge febre ou no primeiro dia de doença. Estudos prévios mostram percentagens mais elevadas de administração de antipiréticos antes do recurso a um serviço de saúde. ${ }^{7}$ Estes dados podem traduzir a fácil acessibilidade aos serviços de urgência que existe em Portugal. ${ }^{1}$

As convulsões foram o principal perigo atribuído à febre não tratada, seguido da possibilidade de lesão cerebral, como observado por outros autores. ${ }^{5-6,8-9}$ Salienta-se que apenas 2,8\% referiu a desidratação como uma possível complicação da febre, percentagem inferior à descrita na literatura. ${ }^{5}$ Apesar de não ter sido alvo de investigação neste estudo, o facto de a maioria dos cuidadores recorrer a um serviço de saúde com medo de uma convulsão febril demonstra a escassez de informação que ainda existe em relação a este tema, o qual deveria ser integrado nos cuidados antecipatórios a realizar ao longo das consultas de saúde infantil.

Relativamente ao local de avaliação escolhido, os autores consideram que pode ter ocorrido um viés dado ter sido um estudo feito no âmbito dos cuidados de saúde primários; salientam, no entanto, a importância da observação pelo médico assistente antes do recurso a um serviço de urgência ou mesmo a uma instituição farmacêutica.

Em suma, perceções e atitudes erradas em relação à febre persistem nos dias de hoje. Ajudar os pais a entender a etiologia da febre, sensibilizar para os benefícios desta em contexto infecioso e informar acerca da administração correta de antipiréticos pode contribuir para diminuir a ansiedade parental e melhorar a prestação de cuidados à criança febril.

\section{REFERÊNCIAS BIBLIOGRÁFICAS}

1. Direção-Geral da Saúde. Processo assistencial integrado da febre de curta duração em idade pediátrica: norma nº 017/2017, de 04/08/2017. Lisboa: DGS; 2017. Existe uma versão atualizada ( $n^{\circ} 14 / 2018$, de 03/08/2018)

2. Schmitt BD. Fever phobia: misconceptions of parents about fevers. Am J Dis Child. 1980;134(2):176-81.

3. Gomide AC, Silva RM, Capanema FD, Gonçalves LA, Rocha RL. How parents deal with the child's fever: influence of beliefs, knowledge, and information sources in the care and management of fever in children systematic review of the literature. Rev Med Minas Gerais. 2014;24(2):175-80.

4. Pestana AP. Conhecimentos e atitudes dos pais perante a febre dos filhos [Children with a fever: parents' knowledge and attitudes]. Rev Port Clin Geral. 2003;19(4):333-43. Portuguese

5. Kelly M, Sahm LJ, Shiely F, O'Sullivan R, McGillicuddy A, McCarthy S. Parental knowledge, attitudes and beliefs regarding fever in children: an interview study. BMC Public Health. 2016;16:540.

6. Rodrigues e Rodrigues L, Monteiro T, Neto T, Rodrigues C. Conhecimentos e atitudes dos pais perante a febre [Knowledges and attitudes 
of parents towards fever]. Saúde Infantil. 2010;32(1):17-21. Portuguese

7. Bertille N, Fournier-Charrière E, Pons $\mathrm{G}$, Chalumeau M. Managing fever in children: a national survey of parents' knowledge and practices in France. PLoS One. 2013;8(12):e83469.

8. Zyoud SH, Al-Jabi SW, Sweileh WM, Nabulsi MM, Tubaila MF, Awang $\mathrm{R}$, et al. Beliefs and practices regarding childhood fever among parents: a cross-sectional study from Palestine. BMC Pediatr. 2013;13:66.

9. Kelly M, Sahm LJ, Shiely F, O'Sullivan R, de Bont EG, Mc Gillicuddy A, et al. Parental knowledge, attitudes and beliefs on fever: a cross-sectional study in Ireland. BMJ Open. 2017;7(7):e015684.

\section{CONFLITO DE INTERESSES}

Os autores declaram não ter quaisquer conflitos de interesse.

\section{ENDEREÇO PARA CORRESPONDÊNCIA}

Ivete Afonso

E-mail: ivete.afonso@hotmail.com

http://orcid.org/0000-0003-4377-3647

Recebido em 23-01-2018

Aceite para publicação em 08-05-2018

\section{ABSTRACT}

\section{PARENTAL KNOWLEDGE, CONCERNS, AND ATTITUDES REGARDING FEVER}

Aim: To characterize parents' knowledge, concerns, and attitudes towards a child with fever, in order to design a future intervention project on this subject for the population.

Type of study: Descriptive and analytic cross-sectional study.

Location: USF Lagoa.

Population: All parents or caregivers of children aged between 29 days and 36 months, enrolled in USF Lagoa.

Methods: Study conducted between September and December 2015, through the distribution of a survey to the caregiver, either by telephone or in person, in case the patient had an appointment during this time period. Data were analyzed with Microsoft Excel $2010{ }^{\circledR}$. This study is reported according to the STROBE guidelines.

Results: A total of 248 parents/caregivers were interviewed. Eighty-three percent of the participants evaluated the temperature in the armpit and $40 \%$ considered fever for values below $37.5^{\circ} \mathrm{C}$. The majority $(54.7 \%)$ chooses paracetamol as the drug of choice, and $30 \%$ knows the adequate dose to be administered. Seizures are considered the main complication associated with fever $(62.1 \%)$. Fifty percent of the participants seek medical care as soon as the fever appears or in the first day of illness, and the primary care center is the care setting of election for half of the respondents.

Conclusions: Wrong perceptions and attitudes towards fever persist nowadays. It is vital to reinforce parents' and caregivers' knowledge on the management and treatment of fever, in order to reduce anxiety and to improve care for febrile children.

Keywords: Antipyretics; Fever; Health information 